\title{
ERRATUM
}

\section{Safety and efficacy of resistance exercise in prostate cancer patients with bone metastases}

P Cormie, RU Newton, N Spry, D Joseph, DR Taaffe and DA Galvão

Prostate Cancer and Prostatic Disease (2015) 18, 196; doi:10.1038/pcan.2015.6

Correction to: Prostate Cancer and Prostatic Diseases (2013) 16, 328-335; doi:10.1038/pcan.2013.22
Following publication of the above article, the authors noticed that in Table 5, the column labels under 12 weeks were reversed. The corrected table appears below. The publisher regrets the errors.

\begin{tabular}{|c|c|c|c|c|c|c|c|c|c|c|c|c|}
\hline \multirow[t]{3}{*}{ Measure } & \multicolumn{4}{|c|}{ Baseline } & \multicolumn{4}{|c|}{12 Weeks } & \multicolumn{4}{|c|}{ Adjusted group differences in mean change over 12 weeks } \\
\hline & \multicolumn{2}{|c|}{ Exercise } & \multicolumn{2}{|c|}{ Usual care } & \multicolumn{2}{|c|}{ Exercise } & \multicolumn{2}{|c|}{ Usual care } & \multirow[t]{2}{*}{ Mean } & \multicolumn{2}{|c|}{$95 \% \mathrm{Cl}$} & \multirow[t]{2}{*}{$\mathrm{P}$} \\
\hline & Mean & s.d. & Mean & s.d. & Mean & s.d. & Mean & s.d. & & & & \\
\hline \multicolumn{13}{|l|}{ Fatigue (MFSI-SF) } \\
\hline Total (score) & 5.2 & 16.8 & 6.0 & 12.3 & 3.8 & 13.7 & 8.8 & 24.9 & -4.2 & -17.6 & 9.2 & 0.521 \\
\hline General (score) & 7.0 & 6.2 & 7.1 & 4.8 & 6.1 & 3.3 & 9.1 & 7.4 & -2.9 & -7.6 & 1.7 & 0.199 \\
\hline Physical (score) & 3.1 & 3.0 & 3.4 & 2.5 & 2.3 & 1.7 & 2.4 & 2.7 & 0.0 & -1.8 & 1.9 & 0.961 \\
\hline Emotional (score) & 3.5 & 3.7 & 3.3 & 3.0 & 4.2 & 4.2 & 3.4 & 6.5 & 0.5 & -3.1 & 4.1 & 0.772 \\
\hline Mental (score) & 4.3 & 3.2 & 4.6 & 3.8 & 4.8 & 2.6 & 6.7 & 6.5 & -1.5 & -4.3 & 1.3 & 0.261 \\
\hline Vigor (score) & 12.7 & 4.5 & 12.4 & 2.5 & 13.3 & 5.5 & 13.1 & 5.7 & -0.1 & -3.4 & 3.2 & 0.931 \\
\hline \multicolumn{13}{|l|}{ Quality of life (SF-36) } \\
\hline Physical functioning (NBS) & 44.2 & 9.0 & 45.0 & 11.4 & 45.8 & 7.8 & 46.5 & 9.4 & 0.0 & -4.2 & 4.2 & 0.996 \\
\hline Role physical (NBS) & 42.9 & 11.5 & 40.7 & 10.8 & 44.7 & 8.1 & 43.4 & 11.1 & 0.0 & -7.2 & 7.2 & 0.998 \\
\hline Bodily pain (NBS) & 48.8 & 8.8 & 49.7 & 10.7 & 45.4 & 8.7 & 48.8 & 8.7 & -2.8 & -9.1 & 3.5 & 0.356 \\
\hline General health (NBS) & 45.6 & 10.0 & 42.4 & 8.6 & 44.5 & 9.8 & 41.7 & 8.6 & 1.9 & -4.1 & 7.9 & 0.508 \\
\hline Vitality (NBS) & 45.8 & 12.7 & 49.3 & 11.3 & 46.1 & 9.1 & 44.5 & 11.9 & 3.7 & -3.7 & 11.1 & 0.303 \\
\hline Social functioning (NBS) & 41.1 & 7.5 & 38.1 & 9.4 & 43.5 & 5.4 & 40.2 & 10.3 & 2.2 & -5.3 & 9.7 & 0.550 \\
\hline Role emotional (NBS) & 37.8 & 8.9 & 36.6 & 9.0 & 34.8 & 10.3 & 37.2 & 11.5 & -3.2 & -12.3 & 5.8 & 0.461 \\
\hline Mental health (NBS) & 50.6 & 10.4 & 50.6 & 7.9 & 52.9 & 8.1 & 50.7 & 13.7 & 2.2 & -6.1 & 10.6 & 0.578 \\
\hline Physical health composite & 45.2 & 11.2 & 45.1 & 10.2 & 45.8 & 8.5 & 45.9 & 9.1 & -0.1 & -4.6 & 4.4 & 0.957 \\
\hline Mental health composite & 44.1 & 10.1 & 43.5 & 7.2 & 43.9 & 11.4 & 42.6 & 12.9 & 2.8 & -5.3 & 11.0 & 0.475 \\
\hline \multicolumn{13}{|l|}{ Psychological distress (BSI-18) } \\
\hline Depression (score) & 2.9 & 4.0 & 1.4 & 2.1 & 3.7 & 6.1 & 3.7 & 5.7 & -2.3 & -5.2 & 0.6 & 0.107 \\
\hline Anxiety (score) & 1.7 & 1.8 & 1.5 & 2.0 & 2.7 & 3.2 & 3.3 & 5.5 & -1.0 & -3.8 & 1.8 & 0.471 \\
\hline Somatization (score) & 2.3 & 2.4 & 2.3 & 3.2 & 2.8 & 2.5 & 1.9 & 2.0 & 1.0 & -1.1 & 3.0 & 0.346 \\
\hline Global severity index (score) & 6.9 & 6.2 & 5.2 & 5.6 & 9.2 & 11.4 & 9.2 & 12.8 & -3.0 & -9.2 & 3.1 & 0.311 \\
\hline
\end{tabular}

Abbreviations: BSI-18, brief symptom inventory; MFSI-SF, multidimensional fatigue symptom inventory-short form; NBS, norm-based score; SF-36, medical

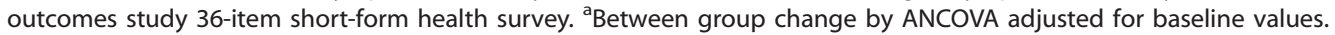

